\title{
Urachal mucinous cystic tumor of low malignant potential in a polymorbid female: a case report and review of the literature
}

\author{
Benjamin Schmeusser ${ }^{1}\left[\right.$ D Joseph Wiedemer ${ }^{1}\left[\right.$ D $\cdot$ Dana Obery $^{1} \cdot$ Kaila Buckley $^{2} \cdot$ Michael Yu $^{1,3}$
}

Received: 17 November 2021 / Accepted: 21 December 2021 / Published online: 15 January 2022

(c) The Author(s) 2022

\begin{abstract}
Neoplasms of the urachus are exceedingly rare, representing $0.17 \%$ of all bladder cancers. The mucinous cystic tumor of low malignant potential (MCTLMP) subtype is particularly rare with just 25 previous cases reported in the literature. Although rare, MCTLMPs are important to identify due to potential devastating complications and good cure rates with surgical removal. We present a 43 year old female with a nuanced constellation of comorbidities and confirmed MCTLMP following a workup for abdominal pain and irritative lower urinary tract symptoms. Notably, this tumor did not change in size over a 3-year course of serial imaging prior to surgical excision. This urachal MCTLMP represents roughly the 26th and one of the smallest of its subtype reported in the literature. This case illustrates the diagnosis and management of this rare urachal MCTLMP. Individual patient medical history, clinical considerations, and neoplasm characteristics are examined. Although rare, the potential for increased malignancy and potential complications necessitates surgical management and further investigation by the academic community.
\end{abstract}

Keywords Case report $\cdot$ Urachal cystadenoma $\cdot$ Uro-oncology $\cdot$ Urachus $\cdot$ Mucinous cystadenoma

\section{Introduction}

The urachus is an embryologic remnant of the allantois, which connects the anterior dome of the bladder to the umbilicus and rarely persists after early infancy. In the event of incomplete atresia, the urachus has the potential to transform into numerous pathologies including fistula, diverticulum, or tumor. Among the potential pathologies of urachal remnants, urachal neoplasms are exceedingly rare and are of significant interest due to their potentially aggressive

Benjamin Schmeusser, Joseph Wiedemer and Dana Obery contributed equally to this work and are considered to be co-first authors.

Benjamin Schmeusser

Schmeusser.2@wright.edu

1 Wright State University Boonshoft School of Medicine, 3640 Colonel Glenn Highway, Dayton, OH 45324, USA

2 Department of Pathology, CORPath/Riverside Methodist Hospital Associates, Columbus, OH, USA

3 Grant Medical Center, Ohio Health Urology, Columbus, OH, USA invasive potential. Invasive mucinous cystadenocarcinomas have a poor prognosis with only $45 \% 5$ year survival rates. $[1,2]$

Isolated cases of urachal neoplasms have presented as pseudomyxoma peritonei and mucosal urinary secretion. Symptomatic presentation varies significantly, but most manifestations include some form of nonspecific abdominal pain [3]. Surgery comprised of excision with partial cystectomy remains the mainstay of care as it is typically curative for noninvasive localized disease [4]. Radiochemotherapeutic intervention has not been studied in randomized multicenter trials and is generally not utilized.

The inner wall of the urachus is lined by epithelium, which may transform into non-glandular, glandular, or mixed neoplasms [4]. Glandular neoplasms are further divided into adenomas, mucinous cystic tumor of low malignant potential (MCTLMP), and adenocarcinoma subtypes [4]. Mucinous cystic tumors, also known as cystadenomas, may be more commonly found in the appendix, ovaries, and pancreas. It is believed that mucinous cystadenocarcinomas can develop from the cystadenomas and MCTLMP [1, 3]. Less than 70 urachal neoplasms have been reported in the literature, among which include just 25 prior known cases of MCTLMP first reported in 2006 [1, 4]. Seven of these reported tumors demonstrated 
calcifications on imaging $[1,5]$. We present the 26th known reporting of MCTLMP.

\section{Case presentation}

A 43 year-old female with a history of newborn gastroschisis, appendectomy, cholecystectomy, colectomy, Roux-en-Y Gastric Bypass, and multiple psychiatric diagnoses had a $\mathrm{CT}$ abdomen/pelvis with contrast that incidentally reported a small diverticulum near the bladder dome. A subsequent CT of the abdomen and pelvis without IV contrast 2 years later once again identified the lesion unchanged in size, although suggested a small bladder saccule, diverticulum, or urachal remnant as possible etiologies. Repeat imaging reports failed to identify this abnormality or found it to be stable. Shortly after, the patient was referred to Urology by her primary care physician due to persistent idiopathic lower abdominal pain following an extensive workup. Symptoms at time of urologic consultation included diffuse low- to-mid abdominal pain with associated nausea, vomiting, anorexia, dysuria, and irritative lower urinary tract symptoms.

Cystoscopic evaluation demonstrated a saccular cystic lesion consistent with a urachal remnant cyst with associated tenderness upon compression. 3 years after initial detection on imaging, an open partial cystectomy for excision of the suspicious region was offered as robotic operation was excluded due to prior abdominal surgical history. Intraoperatively, the urachal remnant was palpable and grossly visible on the anterior bladder wall. Abdominal adhesions were extensive as expected from her significant past abdominal surgical history. A partial cystectomy at the dome of the bladder was performed with careful removal of the involved tissue and without any major intraoperative complications.

Gross examination of the excised specimen revealed a $3.5 \mathrm{~cm}$ area of nodularity believed to represent the actual size of the tumor. Histopathological analysis revealed cystic structures lined by mucinous epithelium with focal areas exhibiting a pseudostratified cyst lining and at least mildto-moderate nuclear atypia, consistent with a mucinous cystadenoma of low malignant potential of the urachus (Figs. 1, 2). Histopathological diagnosis was confirmed with a secondary read by Mayo Clinic Laboratories.

Unfortunately, as of roughly two months status-post surgical excision, the patient has reported no symptomatic relief in the symptoms she believed were caused by the excised urachal remnant.

\section{Discussion}

Interestingly, this patient's history of newborn gastroschisis necessitates consideration of a connection with her subsequent MCTLP. It is unclear if any of the cases in the

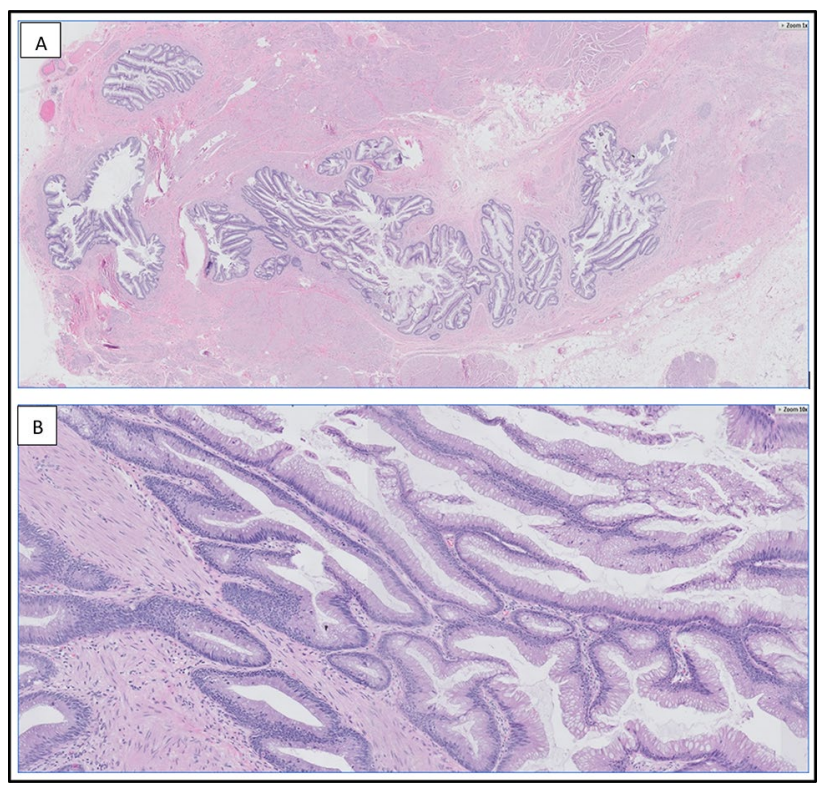

Fig. 1 A, B Histopathologic findings. Multiloculated cystic proliferation composed of a variably complex villous architecture, predominantly lined by a single layer of mucinous epithelium with mild to moderate cytologic atypia. A few mitotic figures are identified. Original magnifications, $\times 1(\mathbf{A}), \times 10(\mathbf{B})$

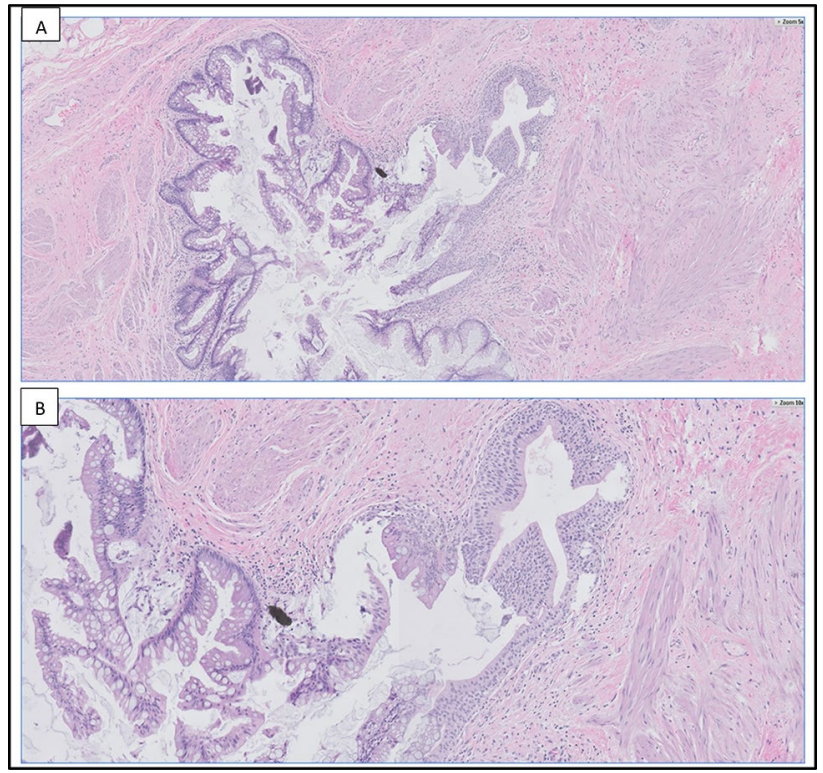

Fig. 2 A, B Histopathologic findings. Mucinous cystic tumor of low malignant potential (MCTLMP). A focus of benign stratified urothelium is visualized on the right, transitioning to atypical mucinous epithelium on the left. Original magnifications, $\times 5(\mathbf{A}), \times 10(\mathbf{B})$

literature had a known history of gastroschisis. Although the specific pathogenesis of gastroschisis is unclear, hypotheses such as mesenchymal failures, amniotic rupture, persistence of umbilical veins, and vitelline artery disruption should be 


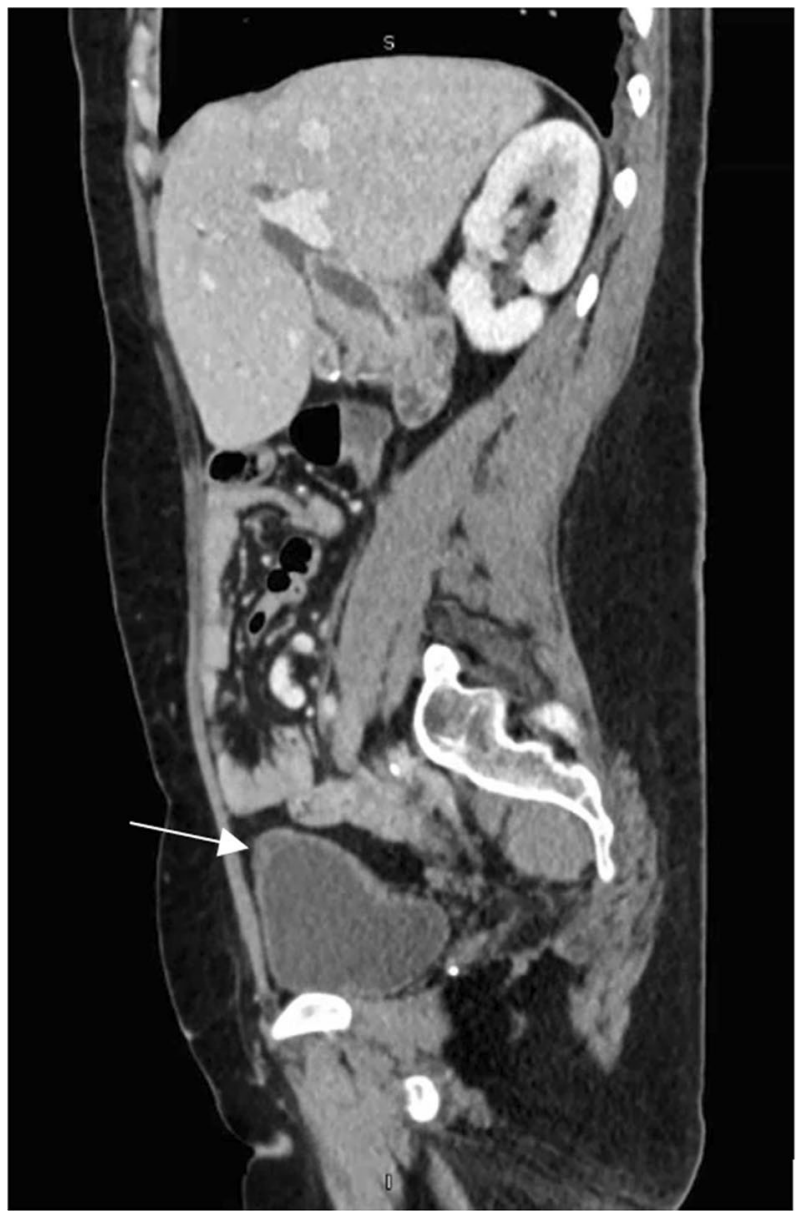

Fig. 3 Sagittal view of non-calcified urachal cystic tumor as seen on CT imaging roughly 2 weeks pre-operative noted with white arrow. Tumor remained unchanged in size on imaging from 3 years prior to excision

considered in the conversation of urachal transformation and carcinogenesis. With relation to the urachus, some hypotheses suggest a connection to ventral folding, ventral structures, and the yolk sac [5]. Among the 25 previous reported cases of MCTLMP [4-8], including this patient, the average tumor size is $4.7 \mathrm{~cm}$. Gender does not appear to be a risk factor for MCTLMP occurrence with 13 of the reported cases observed in females and 12 in males.

Furthermore, this patient's neoplasm was sized at 1.9 $\times 2 \mathrm{~cm}$ on imaging and $3.5 \mathrm{~cm}$ on pathological analysis, differing greatly from reported average size of published mucinous urachal cysts of low malignant potential is $5.4 \mathrm{~cm}(2.0-14 \mathrm{~cm})$ (see Fig. 3) [1]. Given the small size and subtlety of her neoplasm, diagnosis was potentially delayed due to it being missed on imaging and not being palpable on physical exam until her pre-operative cystoscopy. However, given the patient's extensive medical history and postoperative outcome, it is likely that the urachal neoplasm contributed very little to her constellation of symptoms, if at all. This would be consistent with previous cases reported in the literature, in which clinically meaningful symptomatic presentation is relatively rare, especially in the early stages of this disease [9]. Among the previously reported 25 cases of MCTLMP, 11 were incidentally detected while the remaining cases presenting with nonspecific symptoms with the exception of one case that presented with pseudomyxoma peritonei (Table 1). There are no known cases of MCTLMP that have presented with peritoneal carcinomatosis in the medical literature to date. This patient's extensive surgical and medical history must be considered as well as multiple complications and diagnoses such as adhesions, bowel obstructions, gynecological issues, among others, were all likely and more common and probable causes of her symptoms. These issues may have delayed her referral, diagnosis, and surgical treatment and it is fortunate that all potential causes were explored.

An interesting aspect of diagnosing MCTLMP and other urachal neoplasms is that diagnosis is based almost entirely on histologic features [3], as described above and demonstrated in Figs. 2 and 3 for our case. Mucinous cystic tumors are classified similarly to mucinous cystic tumors of the ovary, with MCTLMP being defined as a "cystic tumor with areas of epithelial proliferation, including papillary formation and low-grade atypia [9]." Furthermore, MCTLMP linings are more proliferative than cystadenomas and range from flat to tufted and from pseudopapillary to tubulovillous [9].

Immunohistochemical (IHC) analysis was not completed on initial or confirmatory analyses as it does not appear to add any diagnostic value for urachal neoplasms [3]. A study examining the immunohistochemical expression of urachal carcinomas in search of improved delineation of neoplasms found that urachal carcinomas have an overlapping immunoprofile and therefore offered little clinical benefit [10]. However, in cases presenting as a distantly metastasized cystadenocarcinoma, IHC may be necessary to determine tissue origin. Urachal mucinous cystic tumors stain positively for CK20, CDX2, and CK7 in 100\%, 80\%, and 30\% of the cases, respectively, with negative nuclear $\beta$-catenin, estrogen receptor, and progesterone receptor staining [3].

Given the potential for complications, growth, and increased malignancy, surgical management of mucinous urachal neoplasms is necessary. MCTLMP should be surgically excised with partial cystectomy followed by periodic imaging and cystoscopy to monitor for recurrence. Future cases concerning for urachal neoplasm should include gross imaging and cystoscopy imaging to contribute to the medical literature, improve characterization of these masses, and improve our overall understanding of these neoplasms. Although data is limited, it appears that the prognosis of MCTLMP is excellent following adequate surgical removal. 
Table 1 Summary of previous cases of urachal MCTLMPs

\begin{tabular}{|c|c|c|c|c|c|c|}
\hline Reference & Age & Sex & Size $(\mathrm{cm})$ & Diagnosis & Treatment & Symptoms \\
\hline Shinohara et al. [6] & 54 & M & 6 & MCTLMP & Mass excision/partial cystectomy & Pseudomyxoma peritonei \\
\hline Amin et al. [7] & 48 & $\mathrm{~F}$ & 8 & $\begin{array}{l}\text { MCTLMP } \\
\text { with } \\
\text { intraepi- } \\
\text { thelial } \\
\text { carcinoma }\end{array}$ & $\begin{array}{l}\text { Mass/excision/partial cystectomy and umbi- } \\
\text { lectomy }\end{array}$ & Hematuria, mass \\
\hline Amin et al. [7] & 26 & $\mathrm{~F}$ & 2 & MCTLMP & Mass excision/partial cystectomy & Suprapubic mass \\
\hline Amin et al. [7] & 74 & M & 6.5 & MCTLMP & Excision of tumor and sigmoid colectomy & Incidental finding \\
\hline Amin et al. [7] & 72 & M & 0.8 & MCTLMP & Mass excision/partial cystectomy & Mucusuria \\
\hline Amin et al. [7] & 74 & M & 3 & MCTLMP & Mass excision/partial cystectomy & Hematuria \\
\hline Amin et al. [7] & 50 & $\mathrm{~F}$ & 2.1 & MCTLMP & Mass excision/partial cystectomy & Mass \\
\hline Amin et al. [7] & 45 & M & 3.5 & MCTLMP & Mass excision/partial cystectomy & Right lower quadrant pain/hematuria \\
\hline Amin et al. [7] & 58 & $\mathrm{~F}$ & 1 & MCTLMP & Mass excision/partial cystectomy & Incidental finding \\
\hline Amin et al. [7] & 43 & $\mathrm{~F}$ & 2.5 & MCTLMP & Mass excision/partial cystectomy & Incidental finding \\
\hline Amin et al. [7] & 40 & $\mathrm{~F}$ & 6 & MCTLMP & $\begin{array}{l}\text { Mass/excision/partial cystectomy \& umbilec- } \\
\text { tomy }\end{array}$ & Incidental finding \\
\hline Amin et al. [7] & 80 & $\mathrm{~F}$ & 2.5 & MCTLMP & Mass excision/partial cystectomy & Mucusuria \\
\hline Amin et al. [7] & 37 & $\mathrm{~F}$ & NA & MCTLMP & NA & Incidental finding \\
\hline Amin et al. [7] & 29 & $\mathrm{~F}$ & NA & MCTLMP & NA & Bladder dome nodule \\
\hline Amin et al. [7] & 42 & $\mathrm{~F}$ & 8 & MCTLMP & Mass excision/partial cystectomy & Pelvic mass \\
\hline Amin et al. [7] & 42 & $\mathrm{~F}$ & 6 & MCTLMP & NA & Midline cystic mass \\
\hline Amin et al. [7] & 36 & $\mathrm{~F}$ & NA & MCTLMP & NA & Incidental finding \\
\hline Amin et al. [7] & 39 & M & 6.5 & MCTLMP & $\begin{array}{l}\text { Mass excision/partial cystectomy and umbi- } \\
\text { lectomy }\end{array}$ & Obstruction and umbilical discharge \\
\hline Amin et al. [7] & 57 & M & 2.8 & $\begin{array}{l}\text { MCTLMP } \\
\text { with } \\
\text { intraepi- } \\
\text { thelial } \\
\text { carcinoma }\end{array}$ & Mass excision/partial cystectomy & NA \\
\hline Amin et al. [7] & 77 & $\mathrm{~F}$ & 5.5 & MCTLMP & Mass excision/partial cystectomy & NA \\
\hline Amin et al. [7] & 43 & M & 7 & MCTLMP & $\begin{array}{l}\text { Mass excision/partial cystectomy and umbi- } \\
\text { lectomy }\end{array}$ & Incidental finding \\
\hline Amin et al. [7] & 26 & M & 8 & MCTLMP & Mass excision/partial cystectomy & Urgency, abdominal pain \\
\hline Chahal et al. [8] & 37 & M & 4 & MCTLMP & Partial cystectomy, left hydrocelectomy & Incidental finding \\
\hline Wang et al. [5] & 54 & M & 4 & MCTLMP & $\begin{array}{l}\text { Mass excision/partial cystectomy and umbi- } \\
\text { lectomy }\end{array}$ & Incidental finding \\
\hline Brennan et al. [4] & 67 & M & 9 & MCTLMP & Mass excision/partial cystectomy & Incidental finding \\
\hline Present case (2021) & 43 & $\mathrm{~F}$ & 3.5 & MCTLMP & Mass excision/partial cystectomy & Incidental finding \\
\hline
\end{tabular}

26 cases of urachal MCTLMP have been found in the literature. Table is sorted by date of publication, and obtainable data on age, sex, size, treatment, and symptoms are included

MCTLMP mucinous cystic tumor of low malignant potential, NA Not applicable

\section{Conclusion}

This case illustrates the diagnosis and management of this rare urachal MCTLMP. Individual patient medical history, clinical considerations, and neoplasm characteristics are examined. Although rare, the potential for increased malignancy and potential complications necessitates surgical management and further investigation by the academic community.
Funding Unrestricted grant from the Wright State University Department of Pharmacology and Toxicology.

\section{Declarations}

Conflict of interest Each of the authors declare that they have no known competing financial interests, personal relationships, or any other relevant disclosures that could have appeared to influence the work reported in this paper. 
Open Access This article is licensed under a Creative Commons Attribution 4.0 International License, which permits use, sharing, adaptation, distribution and reproduction in any medium or format, as long as you give appropriate credit to the original author(s) and the source, provide a link to the Creative Commons licence, and indicate if changes were made. The images or other third party material in this article are included in the article's Creative Commons licence, unless indicated otherwise in a credit line to the material. If material is not included in the article's Creative Commons licence and your intended use is not permitted by statutory regulation or exceeds the permitted use, you will need to obtain permission directly from the copyright holder. To view a copy of this licence, visit http://creativecommons.org/licenses/by/4.0/.

\section{References}

1. Wu J, Liu A, Chen A, Zhang P (2017) Urachal borderline mucinous cystadenoma: a rare case report and literature review. Medicine 96(47):e8740

2. Agnihotri AK, Yagnik VD, Agnihotri S, Yagnik B (2020) Mucinous cystadenomas of urachus: a case report and literature review. Urology Ann 12(3):291-294. https://doi.org/10.4103/UA.UA_ 118_19

3. Wang D, Sule N (2018) Mucinous cystadenoma of the urachus and review of current classification of urachal mucinous cystic neoplasms. Arch Pathol Lab Med 143(2):258-263. https://doi. org/10.5858/arpa.2017-0319-RS

4. Brennan K, Johnson P, Curtis H, Arnason T (2019) Urachal mucinous cystic tumor of low malignant potential with concurrent sigmoid colon adenocarcinoma. Case Rep Gastrointest Med 2019:1434838. https://doi.org/10.1155/2019/1434838
5. Wang LL, Liddell H, Tanny ST, Norris B, Appu S, Pan D (2016) Incidental finding of a rare urachal pathology: urachal mucinous cystic tumour of low malignant potential. Case Rep Urol 2016:5764625. https://doi.org/10.1155/2016/5764625

6. Shinohara T, Misawa K, Sano H, Okawa Y, Takada A (2006) Pseudomyxoma peritonei due to mucinous cystadenocarcinoma in situ of the urachus presenting as an inguinal hernia. Int J Clin Oncol 11(5):416-419. https://doi.org/10.1007/s10147-006-0594-1

7. Amin MB, Smith SC, Eble JN et al (2014) Glandular neoplasms of the urachus: a report of 55 cases emphasizing mucinous cystic tumors with proposed classification. Am J Surg Pathol 38(8):1033-1045. https://doi.org/10.1097/pas.0000000000000250

8. Chahal D, Martens M, Kinahan J (2015) Mucinous cystic tumour of low malignant potential presenting in a patient with prior nonseminatous germ cell tumour. Can Urol Assoc J 9(9-10):E750 E753. https://doi.org/10.5489/cuaj.2946

9. Paner GP, Lopez-Beltran A, Sirohi D, Amin MB (2016) Updates in the pathologic diagnosis and classification of epithelial neoplasms of urachal origin. Adv Anat Pathol 23(2):71-83. https:// doi.org/10.1097/pap.0000000000000110

10. Paner GP, McKenney JK, Barkan GA et al (2011) Immunohistochemical analysis in a morphologic spectrum of urachal epithelial neoplasms: diagnostic implications and pitfalls. Am J Surg Pathol 35(6):787-798. https://doi.org/10.1097/PAS.0b013e3182189c11

Publisher's Note Springer Nature remains neutral with regard to jurisdictional claims in published maps and institutional affiliations. 